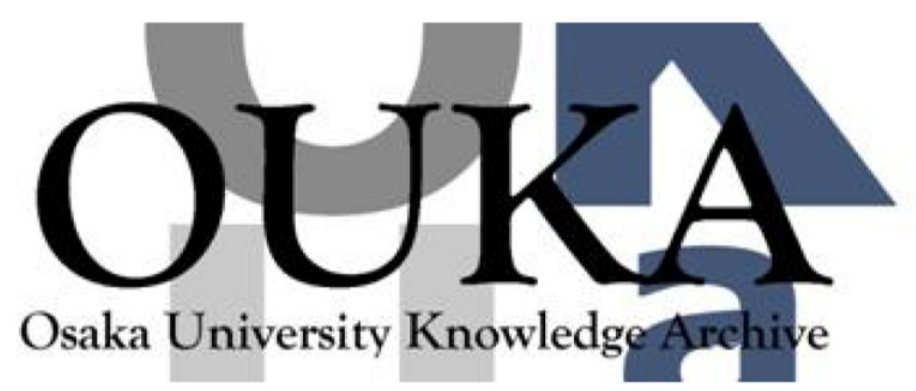

\begin{tabular}{|c|c|}
\hline Title & $\begin{array}{l}\text { Vacuum Bloch-Siegert Shift in Cyclotron } \\
\text { Resonance }\end{array}$ \\
\hline Author (s) & Bamba, Motoaki; Li, Xinwei; Kono, Junichiro \\
\hline \multicolumn{2}{|l|}{ Citation } \\
\hline Issue Date & $2018-09-13$ \\
\hline oaire:version & AM \\
\hline URL & https://hdl. handle. net/11094/70651 \\
\hline rights & $\begin{array}{l}\text { () } 2018 \text { IEEE. Personal use of this material is } \\
\text { permitted. Permission from IEEE must be } \\
\text { obtained for all other uses, in any current or } \\
\text { future media, including reprinting/republishing } \\
\text { this material for advertising or promotional } \\
\text { purposes, creating new collective works, for } \\
\text { resale or redistribution to servers or lists, } \\
\text { or reuse of any copyrighted component of this } \\
\text { work in other works. }\end{array}$ \\
\hline Note & \\
\hline
\end{tabular}

Osaka University Knowledge Archive : OUKA

https://ir. Library. osaka-u. ac. jp/

Osaka University 


\title{
Vacuum Bloch-Siegert Shift in Cyclotron Resonance
}

\author{
Motoaki Bamba ${ }^{1,2}$, Xinwei $\mathrm{Li}^{3}$, and Junichiro Kono ${ }^{3,4,5}$ \\ ${ }^{1}$ Department of Materials Engineering Science, Osaka University, Toyonaka, Osaka 560-8531, Japan \\ ${ }^{2}$ PRESTO, Japan Science and Technology Agency, Kawaguchi, Saitama 332-0012, Japan \\ ${ }^{3}$ Department of Electrical and Computer Engineering, Rice University, Houston, Texas 77005, USA \\ ${ }^{4}$ Department of Material Science and NanoEngineering, Rice University, Houston, Texas 77005, USA \\ ${ }^{5}$ Department of Physics and Astronomy, Rice University, Houston, Texas 77005, USA
}

\begin{abstract}
In a two-dimensional electron gas (2DEG) inside a terahertz (THz) cavity in a perpendicular magnetic field, we observed a clear resonance-frequency shift originating from the counter-rotating coupling between the electrons' cyclotron resonance and $\mathrm{THz}$ cavity modes. This shift can be understood as a vacuum Bloch-Siegert shift, arising from the ultrastrong counter-rotating coupling between the cyclotron-orbiting 2DEG and the vacuum fluctuations of the THz cavity modes. While such a shift has been difficult to observe clearly and is usually neglected under the rotating-wave approximation, here an unambiguous observation was made possible by the broken time-reversal symmetry of the 2DEG in a magnetic field and the use of an ultrahigh-mobility 2DEG, a high-quality-factor cavity, and circularly polarized $\mathrm{THz}$ radiation.
\end{abstract}

\section{INTRODUCTION}

$\mathrm{C}$ oupling of an electromagnetic wave with an electromagnetic polarization in matter is usually investigated under the rotating-wave approximation (RWA), which neglects the counter-rotating coupling of the two oscillating fields. Even when the RWA is invalidated by the use of a high-intensity electromagnetic wave, it is usually difficult to distinguish between corotating and counter-rotating contributions (which induce the optical Stark shift and the Bloch-Siegert shift, respectively), unless the driven material exhibits special selection rules [1].

Even in the limit of a low-intensity electromagnetic wave, the counter-rotating contribution cannot be neglected when the coupling strength is in the ultrastrong coupling regime [2], where the vacuum Rabi splitting is comparable to, or larger than, the resonance frequencies of the electromagnetic wave and oscillating electromagnetic polarization. Counter-rotating coupling can cause fascinating phenomena, such as the appearance of vacuum photons in the ground state of the coupled system [2]. However, the corotating and counterrotating contributions in the ultra-strong coupling regime have never been clearly separated. Here, we report a clear observation of a vacuum Bloch-Siegert shift caused by the ultrastrong counter-rotating coupling of $\mathrm{THz}$ light and matter.

A combination of an ultrahigh-mobility GaAs twodimensional electron gas (2DEG), high- $Q \mathrm{THz}$ cavity photons, and ultrastrong coupling between them led to ultrahigh cooperativity polaritons in the $\mathrm{THz}$ frequency range [3]. As shown in Fig. 1a, in an external static magnetic flux density $B_{\text {stat }}>0$, the cyclotron resonance (CR) of the 2DEG couples with the "+" circularly polarized $\mathrm{THz}$ mode in a corotating manner but with the "-" mode in a counter-rotating manner. As discussed below in detail, when we focus on the "+" circularly polarized mode, we observe resonance frequencies exhibiting the vacuum Rabi splitting (anticrossing) on the positive $B_{\text {stat }}$ side and the vacuum Bloch-Siegert shift (resonance frequency shift) on the negative $B_{\text {stat }}$ side as sketched in Fig. $1 \mathrm{~b}$.

\section{Calculations}

We describe this system starting from the so-called minimalcoupling Hamiltonian:

$\widehat{H}=\int \mathrm{d} \boldsymbol{r}\left[\frac{\epsilon_{0} \epsilon_{\mathrm{cav}}(z) \widehat{\boldsymbol{E}}(z)^{2}}{2}+\frac{\widehat{\boldsymbol{B}}(z)^{2}}{2 \mu_{0}}\right]+\sum_{j=1}^{N} \frac{\left[\widehat{\boldsymbol{\pi}}_{j}+e \widehat{\boldsymbol{A}}\left(z_{0}\right)\right]^{2}}{2 m^{*}}$.

The first two terms represent the energy of the transverse electromagnetic waves. For simplicity, we consider only plane waves perpendicular to the $2 \mathrm{DEG}$ in the $x-y$ plane. The cavity structure is described by the $z$-dependent relative permittivity $\epsilon_{\text {cav }}(z) . \quad \epsilon_{0}$ and $\mu_{0}$ are the vacuum permittivity and permeability, respectively. The oscillating magnetic flux density $\widehat{\boldsymbol{B}}(z)=\boldsymbol{\nabla} \times \widehat{\boldsymbol{A}}(z)$ is described by a vector potential $\widehat{\boldsymbol{A}}(z)$, and the transverse electric field $\widehat{\boldsymbol{E}}(z)=-\widehat{\boldsymbol{\Pi}}(z) /$ $\left[\epsilon_{0} \epsilon_{\text {cav }}(z)\right]$ is described by the conjugate momentum $\widehat{\boldsymbol{\Pi}}(z)$ of $\widehat{A}(z)$. They satisfy $\left[\hat{A}_{\xi}(z), \widehat{\Pi}_{\xi^{\prime}}\left(z^{\prime}\right)\right]=i \hbar \delta_{\xi, \xi^{\prime}} \delta\left(z-z^{\prime}\right) / S$, where $\xi=\{x, y\}$ and $S$ is the area of the $x-y$ plane. The last term in Eq. (1) is the kinetic energy of the 2DEG placed at $z_{0}$. We assume that the width of the 2DEG is negligible compared to the wavelength of the THz wave. $N$ is the number of electrons, and $m^{*}$ is the effective mass. $\widehat{\boldsymbol{\pi}}_{j}=\widehat{\boldsymbol{p}}_{j}+e \boldsymbol{A}_{\text {stat }}$ represents the in-plane momentum $\widehat{\boldsymbol{p}}_{j}$ of the $j$-th electron and the contribution of the static vector potential $\boldsymbol{A}_{\text {stat }}$ giving the static magnetic flux density $\boldsymbol{B}_{\text {stat }}=\boldsymbol{\nabla} \times \boldsymbol{A}_{\text {stat }}=\left(0,0, B_{\text {stat }}\right)^{\mathrm{t}}$.

We rewrite the kinetic energy as $\sum_{j=1}^{N} \hbar \omega_{\text {cyc }}\left(\hat{c}_{j}^{\dagger} \hat{c}_{j}+1 / 2\right)$ by introducing the cyclotron frequency $\omega_{\mathrm{cyc}}=e\left|B_{\mathrm{stat}}\right| / \mathrm{m}^{*}$ and the following operators:

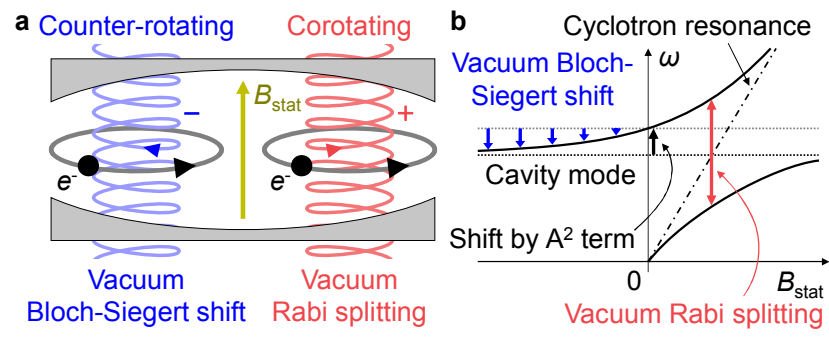

Fig. 1. a, The cyclotron resonance of electrons in an external static magnetic flux density $B_{\text {stat }}$ couples with the "+" circularly-polarized cavity mode in a corotating manner, while it couples with the "-" mode in a counter-rotating manner. The "+" mode shows the vacuum Rabi splitting, while the "-" one shows the vacuum Bloch-Siegert shift. b, Resonance frequencies of the "+" circularly polarized modes are plotted as a function of $B_{\text {stat }}$. We get the vacuum Rabi splitting (anti-crossoing) on the positive $B_{\text {stat }}$ side, while the vacuum Boch-Siegert shift is obtained on the negative $B_{\text {stat }}$ side, where the electrons rotate in the opposite direction as that in Panel a. 


$$
\hat{c}_{j} \equiv \frac{\left(\hat{\pi}_{j, y}+\mathrm{i} \hat{\pi}_{j, x}\right)}{\sqrt{2 m^{*} \hbar \omega_{\text {cyc }}}}, \quad \hat{c}_{j}^{\dagger} \equiv \frac{\left(\hat{\pi}_{j, y}-\mathrm{i} \hat{\pi}_{j, x}\right)}{\sqrt{2 m^{*} \hbar \omega_{\text {cyc }}}} .
$$

They satisfy $\left[\hat{c}_{j}, \hat{c}_{j^{\prime}}^{\dagger}\right]=\delta_{j, j}$. At $B_{\text {stat }}>0, \hat{c}_{j}$ and $\hat{c}_{j^{\prime}}^{\dagger}$ correspond to the lowering and raising operators for the Landau levels, respectively, while the correspondence is reversed at $B_{\text {stat }}<0$. We do not consider Coulomb interactions, since they do not influence linear optical responses due to Kohn's theorem.

In the following calculations, we assume $B_{\text {stat }}>0$ unless specified. For simplicity, we focus only on two degenerate circularly polarized cavity modes with a resonance frequency $\omega_{\text {cav }}$. We describe each mode by an annihilation operator $\hat{a}_{\xi}$ of a photon with a circular polarization $\xi= \pm$. We also focus only on the collective $\mathrm{CR}$ mode interacting with the plane electromagnetic wave. We define its annihilation operator as $\hat{b} \equiv \sum_{j=1}^{N} \hat{c}_{j} / \sqrt{N}$, satisfying $\left[\hat{b}, \hat{b}^{\dagger}\right]=1$. While we should in principle consider the degree of occupation of each Landau level and the coupling enhancement factor $\sqrt{v+1}$ at the $v$-th level, as far as the linear optical responses are concerned, the following Hamiltonian gives the same results [4]:

$$
\begin{aligned}
\widehat{H}=\sum_{\xi= \pm} \hbar \omega_{\text {cav }}\left(\hat{a}_{\xi}^{\dagger} \hat{a}_{\xi}+\frac{1}{2}\right)+\hbar \omega_{\text {cyc }}\left(\hat{b}^{\dagger} \hat{b}+\frac{1}{2}\right) \\
+i \hbar \bar{g}\left[\hat{b}^{\dagger}\left(\hat{a}_{+}+\hat{a}_{-}^{\dagger}\right)-\left(\hat{a}_{-}+\hat{a}_{+}^{\dagger}\right) \hat{b}\right] \\
+\frac{\hbar \bar{g}^{2}}{\omega_{\text {cyc }}}\left(\hat{a}_{+}+\hat{a}_{-}^{\dagger}\right)\left(\hat{a}_{-}+\hat{a}_{+}^{\dagger}\right) .
\end{aligned}
$$

Here, $\bar{g}=g \sqrt{\omega_{\text {cyc }} / \omega_{\text {cav }}}$ is the coupling rate between the collective CR mode and the two cavity modes, where $g=$ $\sqrt{n e^{2} /\left[\epsilon_{0} \epsilon_{\mathrm{cav}}\left(z_{0}\right) m^{*} L_{\mathrm{eff}}\right]}$ is the $B_{\text {stat }}$-independent coupling rate. $n$ is the surface density of the $2 \mathrm{DEG}$, and $L_{\text {eff }}$ is an effective cavity length reflecting the cavity structure $\epsilon_{\text {cav }}(z)$ [4].

The last term in Eq. (3) is the so-called $A^{2}$ term, which comes from the square of the vector potential $\widehat{A}\left(z_{0}\right)$ in the expansion of the kinetic energy $\left[\widehat{\boldsymbol{\pi}}_{j}+e \widehat{\boldsymbol{A}}\left(z_{0}\right)\right]^{2} / 2 m^{*}$ in Eq. (1). The product $\left(e / m^{*}\right) \widehat{\boldsymbol{\pi}}_{j} \cdot \widehat{\boldsymbol{A}}\left(z_{0}\right)$ gives the third term of Eq. (3), which represents the couplings between the CR and the cavity modes. As seen in Eq. (2), a circular motion of electrons corresponds to the lowering operator, and the opposite motion corresponds to the raising one. As a result, the CR couples with the "+" circularly polarized cavity mode in a corotating manner as $\left(\hat{b}^{\dagger} \hat{a}_{+}-\hat{a}_{+}^{\dagger} \hat{b}\right)$, while it couples with the " - " mode in a counter-rotating manner as $\left(\hat{b}^{\dagger} \hat{a}_{-}^{\dagger}-\hat{a}_{-} \hat{b}\right)$. This fact reflects the broken time-reversal symmetry of the electrons in the external magnetic field, as depicted in Fig. 1a, and gives a clear difference between the resonance frequencies of the two circular polarizations $\xi= \pm$, as discussed below.

By solving the Heisenberg equations derived from the Hamiltonian in Eq. (3), we get the following two dispersion relations determining the eigenfrequencies $\omega$ of the system:

$$
\begin{aligned}
\frac{\omega_{\text {cav }}^{2}}{\omega^{2}} & =1-\frac{2 g^{2}}{\omega^{2}}-\frac{2 g^{2}}{\omega\left(\omega-\omega_{\text {cyc }}\right)} \frac{\omega_{\text {cyc }}}{\omega} \\
& =1-\frac{2 g^{2}}{\omega\left(\omega-\omega_{\text {cyc }}\right)},
\end{aligned}
$$

$$
\begin{aligned}
\frac{\omega_{\text {cav }}^{2}}{\omega^{2}} & =1-\frac{2 g^{2}}{\omega^{2}}+\frac{2 g^{2}}{\omega\left(\omega+\omega_{\text {cyc }}\right)} \frac{\omega_{\text {cyc }}}{\omega} \\
& =1-\frac{2 g^{2}}{\omega\left(\omega+\omega_{\text {cyc }}\right)} .
\end{aligned}
$$

The left-hand side of both equations is proportional to $(k / \omega)^{2}$, where $k \equiv \omega_{\text {cav }} \sqrt{\epsilon_{\text {cav }}\left(z_{0}\right)} / c \approx \pi / L_{\text {eff }}$ is the confinement wavenumber of the THz wave in the cavity and $c$ is the speed of light in vacuum. The right-hand side corresponds to the relative permittivity [divided by $\epsilon_{\text {cav }}\left(z_{0}\right)$ ]. The second term in Eqs. (4b) and (5b) is the electric susceptibility $\mathrm{i} \sigma_{ \pm}(\omega) /$ $\left[\epsilon_{0} \epsilon_{\text {cav }}\left(z_{0}\right) \omega L_{\text {eff }}\right]$, reflecting the optical conductivity $\sigma_{ \pm}(\omega)=$ (ine $\left.e^{2} / m^{*}\right) /\left(\omega \mp \omega_{\text {cyc }}\right)$ of the $2 \mathrm{DEG}$ for " \pm ” circularly polarized THz waves. The factor $\left(\omega-\omega_{\text {cyc }}\right)^{-1}$ comes from the corotating coupling $e^{-\mathrm{i}\left(\omega-\omega_{\mathrm{cyc}}\right) t}$ between the CR and the "+" $\mathrm{THz}$ wave, while $\left(\omega+\omega_{\text {cyc }}\right)^{-1}$ comes from the counterrotating coupling $e^{-\mathrm{i}\left(\omega+\omega_{\text {cyc }}\right) t}$ between the CR and the "-" THz wave. The latter contribution is neglected under the RWA.

\section{RESULTS}

When we focus on the case of $B_{\text {stat }}>0$, Eq. (4b) gives two positive $\omega$ solutions (upper and lower cavity polaritons), showing an anticrossing behavior between the CR and cavity frequencies, as plotted by the solid blue line in Fig. 2a. These coupled corotating modes are excited by the "+" THz radiation, conserving the angular momentum. At $B_{\text {stat }}=B_{0}$ giving $\omega_{\text {cyc }}=\omega_{\text {cav }}$, the frequency splitting is approximately expressed as $2 g$, which is the exact value in the limit of $g \ll$ $\omega_{\text {cav }}, \omega_{\text {cyc }}$. This splitting does not depend on the intensity of the incident $\mathrm{THz}$ beam but reflects the vacuum fluctuations $\left\langle\hat{A}_{\xi} \hat{A}_{\xi}\right\rangle=\hbar /\left[\epsilon_{0} \epsilon_{\text {cav }}\left(z_{0}\right) m^{*} L_{\text {eff }}\right]=\hbar g^{2} /\left(n e^{2}\right)$ of the $\mathrm{THz}$ cavity modes. This splitting is called the vacuum Rabi splitting.

On the other hand, Eq. (5b) gives one positive $\omega$ solution without any splitting, as plotted by the red dashed line on the positive $B_{\text {stat }}$ side in Fig. 2a. This corresponds to the cavity
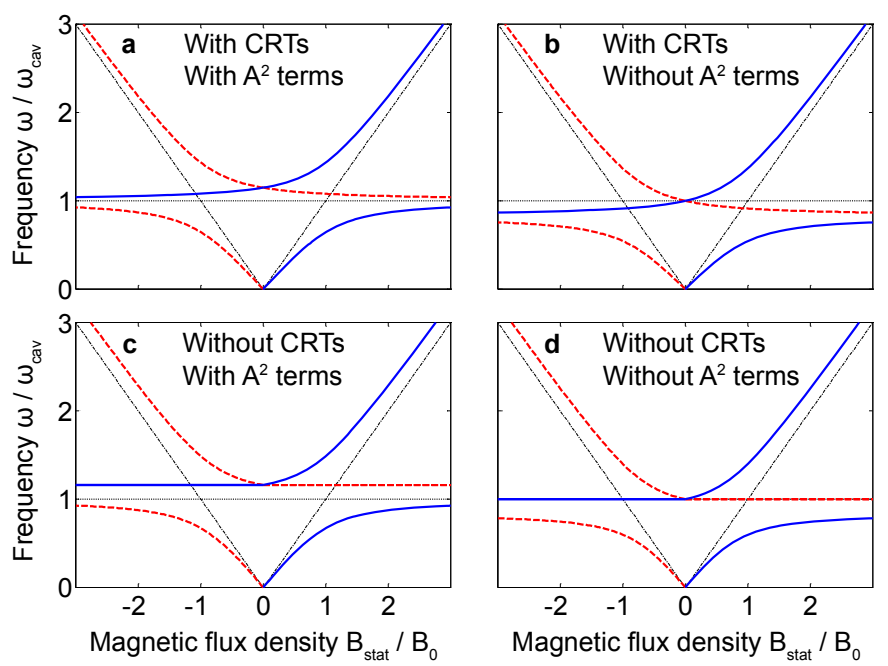

Fig. 2. Eigenfrequencies $\omega$ verus static magnetic flux density $B_{\text {stat }}$, a, with counter-rotating terms (CRTs) and $A^{2}$ terms, b, with CRTs but without $A^{2}$ terms, $\mathbf{c}$, without CRTs but with $A^{2}$ terms, and $\mathbf{d}$, without CRTs and $A^{2}$ terms. $B_{0}$ gives $\omega_{\text {cyc }}=\omega_{\text {cav }}$. The blue solid and red dashed curves represent the "+" and "-" circularly polarized modes, respectively. The black dotted and dasheddotted lines are the cavity and CR frequencies, respectively. Parameters: $g=$ $0.4 \omega_{\text {cav }}$. 
mode that couples with the $\mathrm{CR}$ in the counter-rotating manner, i.e., the electric susceptibility is proportional to $(\omega+$ $\left.\omega_{\text {cyc }}\right)^{-1}$ on the right-hand side of Eq. (5b). This counter-rotating mode is excited by the "-" $\mathrm{THz}$ radiation, conserving the angular momentum. In the limit of $B_{\text {stat }} \rightarrow \infty$, its eigenfrequency asymptotically reaches $\omega_{\text {cav }}$ together with the lower corotating mode since the electric susceptibilities in Eqs. $(4 \mathrm{~b})$ and $(5 \mathrm{~b})$ become negligible $\left(\omega_{\text {cyc }}\right.$ becomes far away from $\left.\omega_{\text {cav }}\right)$. The eigenfrequencies of the counter-rotating and upper corotating modes coincide with each other at $B_{\text {stat }}=0\left(\omega_{\text {cyc }}=\right.$ 0 ) since the coupling rate $\bar{g}=g \sqrt{\omega_{\text {cyc }} / \omega_{\text {cav }}}$ becomes zero and the two circularly polarized $\mathrm{THz}$ waves couple with the 2DEG in the same manner (the time-reversal symmetry is not broken). In the case of $B_{\text {stat }}<0$, the " + " and "-" circularly polarized modes couple with the $\mathrm{CR}$ in the counter- and corotating manner, respectively, since the electrons rotate in the opposite direction to that at $B_{\text {stat }}>0$.

The electric susceptibilities in fact include two contributions described by the last two terms in Eqs. (4a) and (5a). The last terms come from the corotating and counter-rotating couplings between the $\mathrm{CR}$ and cavity modes. On the other hand, the second last term is the contribution of the $A^{2}$ term. It gives a frequency shift of the cavity mode as $\omega_{\text {cav }}^{\prime}=\sqrt{\omega_{\text {cav }^{2}}+2 g^{2}}$, which is equivalent to the solution of Eqs. (4) and (5) at $B_{\text {stat }}=$ $0\left(\omega_{\text {cyc }}=0\right)$ because the coupling rate $\bar{g}=g \sqrt{\omega_{\text {cyc }} / \omega_{\text {cav }}}$ becomes zero while the coefficient $\bar{g}^{2} / \omega_{\text {cyc }}=g^{2} / \omega_{\text {cav }}$ of the $A^{2}$ term remains and does not depend on $B_{\text {stat }}$. In Fig. $2 \mathrm{~b}$, we plot the eigenfrequencies without the contribution of the $A^{2}$ term. The cavity frequency $\omega_{\text {cav }}$ looks red-shifted as $\sqrt{\omega_{\text {cav }}^{2}-2 g^{2}}$, i.e., the eigenfrequencies of the counterrotating modes becomes $\omega=\omega_{\text {cav }}$ at $B_{\text {stat }}=0$ and $\omega \rightarrow$ $\sqrt{\omega_{\text {cav }}^{2}-2 g^{2}}$ in the limit of $B_{\text {stat }} \rightarrow \pm \infty$.

When we apply the RWA in the Heisenberg equations, the last term is eliminated in Eq. (5a) while the contribution of the $A^{2}$ term remains. As a result, as we plotted in Fig. 2c, the eigenfrequencies of the counter-rotating modes are no longer shifted by the change in $B_{\text {stat }}$. We call this red-shift originating from the counter-rotating coupling a vacuum Bloch-Siegert shift. Its shift does not depend on the intensity of the incident $\mathrm{THz}$ beam but reflects the vacuum fluctuations $\left\langle\hat{A}_{\xi} \hat{A}_{\xi}\right\rangle=$ $\hbar g^{2} /\left(n e^{2}\right)$ of the THz cavity modes. The maximum shift is obtained in the limit of $B_{\text {stat }} \rightarrow \pm \infty$ and compensate the shift by the $A^{2}$ term, i.e., $\sqrt{\omega_{\text {cav }}^{2}+2 g^{2}}-\omega_{\text {cav }}$. When we eliminate the contributions of both the counter-rotating terms and the $A^{2}$ terms, the eigenfrequencies of the counter-rotating modes completely agree with the bare cavity frequency $\omega_{\text {cav }}$, as shown in Fig. 2d.

The resonance frequencies that can be obtained experimentally are those in Fig. 2a. The blue-shift of the cavity frequency by the $A^{2}$ term can be extracted from the difference between the resonance frequencies of the counter-rotating modes at $B_{\text {stat }}=0$ and in the limit of $B_{\text {stat }} \rightarrow \infty$ (or frequency difference between the upper corotating mode at $B_{\text {stat }}=0$ and lower corotating one in the limit of $B_{\text {stat }} \rightarrow \infty$ ). The vacuum Bloch-Siegert shift can be extracted as the frequency shift of the counter-rotating mode from that at $B_{\text {stat }}=0$, when CR-cavity coupled systems show a sufficiently high cooperativity parameter.

\section{SUMMARY}

The origin of the unambiguous separation of the contributions of the $A^{2}$ term, corotating and counter-rotating terms can be traced back to the broken time-reversal symmetry of the $2 \mathrm{DEG}$ in the external magnetic flux density $B_{\text {stat. In a }}$ general system without special selection rules of optical excitations in the absence of $B_{\text {stat }}$, the two circularly polarized cavity modes independently couple with two circularly polarized electric polarizations. In this case, each eigenfrequency includes all the contributions of the $A^{2}$ term, corotating and counter-rotating couplings. In contrast, in the presence of $B_{\text {stat }}$, the time-reversal symmetry of electrons is broken. As shown in Eq. (2), a circular motion of electrons corresponds to the lowering operator, and the other motion corresponds to the raising one in the present $2 \mathrm{DEG}$ system. As a result, the CR couples with a circularly polarized mode in the corotating manner but with the other mode in the counterrotating manner, and the contributions of these couplings can be separated by controlling the circular polarization of the incident $\mathrm{THz}$ beam. The contribution of the $A^{2}$ term can also be separated in the present system since the coupling rate $\bar{g}=$ $g \sqrt{\omega_{\text {cyc }} / \omega_{\text {cav }}}$ becomes zero at $B_{\text {stat }}=0$ while the coefficient of the $A^{2}$ term remains and does not depend on $B_{\text {stat }}$.

In the regime of high cooperativity and ultrastrong coupling between the 2DEG CR and THz cavity modes, the counterrotating contribution can be distinguished clearly, as the appearance of a vacuum Bloch-Siegert shift. In addition to the counter-rotating contribution generating virtual photons [2], we can also distinguish the photon-field self-interaction frequency shift by the so-called $A^{2}$ term, which prevents the superradiant phase transition [5]. Our findings provide much significant new insight into the above-mentioned three contributions in general systems in the ultrastrong coupling regime.

\section{ACKNOWLEDGEMENTS}

J.K. acknowledges support from the Army Research Office (grant W911NF17-1-0259) for terahertz magneto-spectroscopy measurements and the National Science Foundation (grant DMR-1310138) for cavity fabrication. M.B. acknowledges support from JST PRESTO (grant JPMJPR1767), KAKENHI (grant 26287087), and ImPACT Program of Council for Science, Technology and Innovation (cabinet office, government of Japan).

\section{REFERENCES}

[1]. E. J. Sie, C. H. Lui, Y.-H. H. Lee, L. Fu, J. Kong, and N. Gedik, "Large, valley-exclusive Bloch-Siegert shift in monolayer $\mathrm{WS}_{2}$," Science, vol. 355, pp. 1066-1069, March, 2017.

[2]. C. Ciuti, G. Bastard, and I. Carusotto, "Quantum vacuum properties of the intersubband cavity polariton field," Physical. Review B, vol. 72, p. 115303, September, 2005.

[3]. Q. Zhang, M. Lou, X. Li, J. L. Reno, W. Pan, J. D. Watson, M. J. Manfra, and J. Kono, "Collective non-perturbative coupling of 2D electrons with highquality-factor terahertz cavity photons", Nature Physics, vol. 12, pp. 10051011, August, 2016.

[4]. X. Li, M. Bamba, Q. Zhang, S. Fallahi, G. C. Gardner, W. Gao, M. Lou, K. Yoshioka, M. J. Manfra, and J. Kono, "Vacuum Bloch-Siegert Shift in Landau Polaritons with Ultrahigh Cooperativity", Nature Photonics, vol. 12, pp. 324329, June, 2018.

[5]. K. Rzążewski, K. Wódkiewicz, and W. Żakowicz, "Phase Transitions, Two-Level Atoms, and the $A^{2}$ Term," Physical Review Letters, vol. 35, pp. 432 434, August, 1975. 